\section{Genomics in black and white (and green)}

\author{
Principles of Genome Analysis and Genomics \\ (3rd edn) \\ SB Primrose and RM Twyman \\ Blackwell Science Ltd, Oxford; 2003. 263 pp. \\ $£ 29.50$, paperback. ISBN 1-40510-120-2
}

Heredity (2004) 92, 598. doi:10.1038/sj.hdy.6800473

\section{Reviewed by John Armour}

What do we mean by 'Genomics'? This book gives a pretty good answer and one that makes the subject accessible at an impressively advanced level while still assuming very little prior knowledge from the reader. The book professes to be aimed at 'advanced undergraduates', and it would be nice to think that some undergraduates are advanced enough to make use of it! My guess is that in real life, larger numbers of postgraduates than undergraduates will turn to it for an introduction to the world of genome analysis.

Whether it will generally be to the taste of Heredity readers is a different matter: appropriately enough for the early 21 st century, some might argue, there is a lot about genomes but precious little genetics. The book starts with an appropriate exposition of the big themes how genome analysis became possible and why it might be useful. After human linkage mapping with RFLPs appears in Chapter 1 as an interesting historical detail, the book begins its explanation of genome analysis entirely from the point of view of physical mapping. In this context, the real genetics belongs in the final chapter on 'applications of genome analysis and genomics'.

As in the many other excellent offerings from Sandy Primrose and his coauthors, most notably the classic Principles of Gene Manipulation, this book is at its strongest in identifying the fundamental principles underlying the subject and then in the careful explanation of detail where detail matters. Even where this requires quite a lengthy treatment, it takes care to give clear answers to the question 'but how does it actually work?' so often neglected by students and so important in understanding modern approaches to genome analysis. For example, we are given a careful explanation of how Serial Analysis of Gene Expression (SAGE) libraries are constructed, and therefore can appreciate what makes them so clever and so powerful. Similarly, the details of many procedures, such as chromosome flow-sorting, YAC cloning, radiation hybrid mapping, pyrosequencing, AFLPs and constructing recombinant inbred lines of which a general understanding is good, but a more detailed understanding is better - are carefully and accurately presented. It is for this wealth of (often quite technical) detail, on which the authors have clearly worked hard, that I think the book is well worth its price.

One of the book's chief strengths leads it into unavoidable weakness; in expounding the general principles of genome analysis, we tend to hear about the genomes themselves almost as mere illustrations of the generalities. It would be a tall order indeed to have woven in detailed information about the results of genome analysis, and perhaps inevitably I found myself thinking that anyone looking to understand any one genome in any depth will have to look elsewhere.

An obvious danger in writing about such a fastmoving subject is that the book will age rapidly, as new information and conclusions qualify its message. The authors appear to have provided some insurance against this, both in using many referenced works from the 'classic original' rather than the 'recent update' category and (where up-to-date information is most important) in providing web references. Best of all, many of the most useful references given at the end of each chapter come with a short summary to help the reader's judgement in choosing appropriate background reading.

Throughout the book the standards of accuracy and attention to detail are exemplary. I found only a single minor typographical error and only one example of a cited reference missing from the reference list. The book benefits from the many diagrams illustrating the principles of methods, but I found them generally lacking in richness and most especially lacking in colour. While it would be quibbling indeed to expect every one of the many diagrams to be lavishly crafted, a little more detail in the figures would allow shorter and clearer descriptions in the text, for example in explaining massively parallel signature sequencing (MPSS). Apart from the visually appealing cover, all the illustrations in the book use a colour scheme drawn from different shades of black, white and green. Whether this was a misguided attempt by the authors to convey serene simplicity in the face of complex subject matter or a misguided preoccupation on the part of the publishers with production costs, I think it is misguided - as it becomes obvious in the attempts to explain fluorescent label ratios on microarrays in this restricted colour scheme. With more detailed and colourful diagrams this would have been a better book. As it is, the quality of the authors' work ensures that it is still very good indeed and deserves to be a widely popular resource for newcomers to genome analysis.

J Armour Institute of Genetics, University of Nottingham, Queen's Medical Centre, Nottingham NG7 2UH, UK E-mail: john.armour@nottingham.ac.uk 